\title{
The thermo-visco-plastic instability analysis of saturated soil
}

\author{
X.B. $\mathrm{Lu}$ \\ Institute of Mechanics, Chinese Academy of Sciences, Bejing 100080, People's Republic of China
}

Received 10 November 1999; accepted 27 March 2000

\begin{abstract}
A theoretical analysis of instability of saturated soil is presented considering the simple shearing of a heat conducting thermo-visco-plastic material. It is shown that the instability is mainly the consequence of thermal softening which overcomes the strain hardening and the other type of instability is controlled by strain softening. The effects of other factors such as permeability to the instability are discussed in this paper. (C) 2001 Elsevier Science Ltd. All rights reserved.
\end{abstract}

Keywords: Saturated soil; Shear load; Instability; Perturbation method

\section{Introduction}

The instability of soil foundation has been the focus of study. Researchers have done lots of experiments and analysis trying to seek for the criterion of soil instability in order to evaluate the safety of foundations under vibration and to avoid instability. Theoretical contributions are related mainly to stability and bifurcation analysis of diffused and localized failure models [1-3]. Typically, the stability problem is formulated by considering small perturbations in field variables (e.g. displacement). Classical continuum approach leads, in this case, to the ordinary diffusion equation for the perturbation in stresses [1,2]. Alternatively, the localization in fluid-infiltrated soil may be considered as a bifurcation problem [4]. The experimental evidence on deformation instabilities and the failure models comes from conventional triaxial $[5,6]$ as well as plain strain biaxial tests [7]. The results

E-mail address: xblu@imech.ac.cn (X.B. Lu). indicated that the uniform response is often followed by the onset of a diffused, nonhomogeneous deformation model, after which distinct shear bands form. The problem is often discussed under inertia-free conditions and without temperature effect $[1,8,9]$. Nevertheless, the temperature has an important role under some conditions [10-12]. Therefore, in the present paper an attempt is made to discuss the thermo-visco-plastic instability under simple shear.

\section{The mathematical model}

\subsection{Some assumptions}

In order to clarify the problem, some assumptions are presented here:

(1) The density of water and gains is constant, which means $\rho_{\mathrm{s}}=$ const., $\rho_{\mathrm{w}}=$ const.

(2) The deformation can only occur in one direction but may have a gradient in the other direction. 
The geometrical configuration and the deformation can be expressed as follows:

$x=X$,

$y=u(X, Y)+Y$,

$z=Z$.

The constitutive equations can be expressed as follows [10-12] under shear load:

$\sigma_{\mathrm{e} x}=f_{1}(\gamma, \dot{\gamma}, \theta)$

$\sigma_{\mathrm{e} y}=f_{2}(\gamma, \dot{\gamma}, \theta)$

$\tau=f_{3}(\gamma, \dot{\gamma}, \theta)$,

in which $\sigma_{\mathrm{e} x}, \sigma_{\mathrm{e} y}$ are effective stresses in $x$ and $y$ directions, respectively, $\tau$ is the shear stress, $\gamma$ the shear strain, $\dot{\gamma}$ the shear strain ratio, and $\theta$ the temperature.

The relationship between the plastic work $W_{\mathrm{p}}$ and the heat $q$ produced by it is as follows:

$q=C_{1} W_{\mathrm{p}}$

and because the elastic deformation energy is much smaller than that due to plastic deformation, the former may be neglected. Thus,

$W_{\mathrm{p}}=\int \sigma_{i j} \dot{\varepsilon}_{i j} \mathrm{~d} t$ law

The heat conduction is governed by Fourier's

$h_{i}=-\lambda \theta_{i} \quad(i=x, y)$

in which $h_{i}$ are the components of heat flux, and $\lambda$ is the thermal conductivity. Therefore, the energy equation becomes

$C_{1} \dot{W}_{\mathrm{p}}=\rho c_{v} \dot{\theta}-\lambda \Delta \theta$

in which $\Delta$ is the Laplace operator. In the case concerned,

$C_{1} \tau \frac{\partial \gamma}{\partial t}=\rho c_{v} \frac{\partial \theta}{\partial t}-\lambda \frac{\partial^{2} \theta}{\partial y^{2}}$,

where $C_{1}$ is a parameter and $C_{1}=C_{1}(p)$.

\subsection{The equilibrium equations}

The momentum equations are as follows $[13,14]$ :

$$
\begin{aligned}
\rho_{\mathrm{s}} \frac{\partial^{2} u_{\mathrm{s} x}}{\partial t^{2}}+\frac{\partial \sigma_{\mathrm{e} x}}{\partial x}-\frac{\partial \tau}{\partial y}= & K n^{2} \frac{\partial}{\partial t}\left(u_{\mathrm{w} x}-u_{\mathrm{s} x}\right), \\
\rho_{\mathrm{s}} \frac{\partial^{2} u_{\mathrm{s} y}}{\partial t^{2}}+\frac{\partial \sigma_{\mathrm{e} y}}{\partial y}-\frac{\partial \tau}{\partial x}= & K n^{2} \frac{\partial}{\partial t}\left(u_{\mathrm{w} y}-u_{\mathrm{s} y}\right) \\
& -(1-n) \rho_{\mathrm{s}} g,
\end{aligned}
$$

where $u_{\mathrm{w} x}, u_{\mathrm{w} y}$ are displacements of pore water in two directions and $u_{\mathrm{s} x}, u_{\mathrm{s} y}$ are displacements of solid phase in two directions, $n$ is the porosity, $K=\mu / k$, where $k$ is the Darcy permeability and $\mu$ is the viscosity.

Differentiating Eq. (8) in $x$ and $y$ directions, respectively and then adding them, the first governing equation is obtained as follows:

$(1-n) \rho_{\mathrm{s}} \frac{\partial^{2} \gamma}{\partial t^{2}}-\frac{\partial^{2} \tau}{\partial y^{2}}=-K n^{2} \frac{\partial \gamma}{\partial t}$

Now, the control equations can be rewritten as

$$
\begin{aligned}
& C_{1} \tau \frac{\partial \gamma}{\partial t}=\rho_{\mathrm{s}} c_{v} \frac{\partial \theta}{\partial t}-\lambda \frac{\partial^{2} \theta}{\partial y^{2}}, \\
& \rho_{\mathrm{s}} \frac{\partial^{2} \gamma}{\partial t^{2}}-\frac{\partial^{2} \tau}{\partial y^{2}}=-K n^{2} \frac{\partial \gamma}{\partial t} .
\end{aligned}
$$

The analytical solutions of these equations are difficult to seek for because of the non-linearity. It has been shown by experiments and computation that the soil deformation develops from slow to fast, that means, from stable state to one of instability.

Now, there are three points should be emphasized: firstly, examing all of the assumptions, Eq. (10) can deal with large shear deformation, because no limitation of the shear deformation has been introduced. Secondly, the first one of Eq. (10) is a wave equation but the right side of the second is a typical diffusion equation, this two types of different phenomenon are coupled through the term $C_{1} \tau \dot{\gamma}$. This is the distinctive feature of the phenomenon under consideration. Finally, Eq. (10) is obviously non-linear.

Since the aim of this paper is to seek for the condition under which a smooth deformation 
process changes into catastrophe, the perturbation method, which is widely used in the analysis of fluid dynamics [15], is adopted here. Hence, a smooth developing deformation state $\gamma_{0}, \tau_{0}, p_{0}$ is taken as the base state which is a solution of Eq. (10). When perturbation has been applied on the control equations, we will be able to analyze the factors and conditions of instability.

\section{Perturbation analysis $[16]$}

To seek for the point deformation implies a shift from smooth to catastrophic, we study the solutions in the following form:

$\gamma=\gamma_{0}+\gamma^{\prime}, \quad\left|\gamma^{\prime}\right| \ll\left|\gamma_{0}\right|$,

$\theta=\theta_{0}+\theta^{\prime}, \quad\left|\theta^{\prime}\right| \ll\left|\theta_{0}\right|$,

where $\gamma_{0}, \theta_{0}$ is a solution Eq. (10), and

$\gamma^{\prime}=\gamma^{*} \mathrm{e}^{\alpha t+\mathrm{i} \beta x}, \quad \theta^{\prime}=\theta^{*} \mathrm{e}^{\alpha t+\mathrm{i} \beta x}$.

$\alpha, \beta$ are, respectively, the frequency and the wave number.

Differentiating the constitutive relations (2), we obtain

$\mathrm{d} \tau=R_{0} \mathrm{~d} \gamma-Q_{0} \mathrm{~d} \theta+H_{0} \mathrm{~d} \dot{\gamma}$

in which

$R_{0}=\left(\frac{\partial \tau}{\partial \gamma}\right)_{0}, \quad Q_{0}=-\left(\frac{\partial \tau}{\partial \theta}\right)_{0}, \quad H_{0}=\left(\frac{\partial \tau}{\partial \dot{\gamma}}\right)_{0}$.

Therefore,

$\tau^{*}=R_{0} \gamma^{*}-Q_{0} \theta^{*}+\alpha H_{0} \gamma^{*}$.

Substituting Eqs. (11), (12) and (15) into Eq. (10), the homogeneous system of equations is obtained as follows:

$$
\begin{aligned}
& {\left[\rho_{\mathrm{s}} \alpha^{2}+\beta^{2}\left(R_{0}+\alpha H_{0}\right)+K n_{0}^{2} \alpha\right] \gamma^{*}-\beta^{2} Q_{0} \theta^{*}=0,} \\
& \left(C_{1} \tau_{0} \alpha+K \dot{\gamma}_{0}\left(R_{0}+\alpha H_{0}\right)\right) \gamma^{*} \\
& \quad-\left(C_{1} \dot{\gamma}_{0} Q_{0}+\rho_{\mathrm{s}} c_{v} \alpha+\lambda \beta^{2}\right) \theta^{*}=0 .
\end{aligned}
$$

As we all know, the determinant of the coefficients should be equal to zero if the system has solutions, which leads to

$\rho_{\mathrm{s}}^{2} c_{v} \alpha^{3}+A_{1} \alpha^{2}+A_{2} \alpha+\lambda R_{0} \beta^{4}=0$,

where

$A_{1}=\left(\rho_{\mathrm{s}} c_{v} H_{0}+\rho_{\mathrm{s}} \lambda\right) \beta^{2}+\rho_{\mathrm{s}} C_{1} \dot{\gamma}_{0} Q_{0}+\rho_{\mathrm{s}} c_{v} K n_{0}^{2}$,

$$
\begin{aligned}
A_{2}= & \lambda H_{0} \beta^{4}+K n_{0}^{2} \lambda \beta^{2}+K n_{0}^{2} C \dot{\gamma}_{0} Q_{0}+\rho_{\mathrm{s}} c_{v} \beta^{2} R_{0} \\
& -C_{1} \tau_{0} Q_{0} \beta^{2} .
\end{aligned}
$$

It is a spectral equation. If $\alpha$ has a positive real root, instability is possible.

Now, we can give the dimensionless form of Eq. (17), using the next dimensionless variables

$$
\begin{aligned}
& \alpha=\frac{c_{v} R_{0}}{\lambda} \bar{\alpha}, \quad \beta^{2}=\frac{\rho_{\mathrm{s}} c_{v}^{2} R_{0}}{\lambda^{2}} \bar{\beta}^{2}, \\
& A=\frac{c_{v} H_{0}}{\lambda}, \quad B=\frac{C_{1} \tau_{0} Q_{0}}{\rho_{\mathrm{s}} c_{v} R_{0}} \\
& C=\frac{C_{1} \lambda \dot{\gamma}_{0} Q_{0}}{\rho_{\mathrm{s}} c_{v}^{2} R_{0}}, \quad D=\frac{K n_{0}^{2} \lambda}{\rho_{\mathrm{s}} c_{v} R_{0}} .
\end{aligned}
$$

Then, the spectral equation (17) can be reduced to the following form:

$$
\begin{aligned}
\bar{\alpha}^{3} & +\left[\bar{\beta}^{2}+C+A \bar{\beta}^{2}+D\right] \bar{\alpha}^{2} \\
& +\left[(1-B+D) \bar{\beta}^{2}+A \bar{\beta}^{4}+D C\right] \bar{\alpha}+\bar{\beta}^{4}=0 .
\end{aligned}
$$

It is obvious that this equation has two extreme situations:

(i) For long wavelength $(\beta \rightarrow 0)$, Eq. (19) has two solutions

$\bar{\beta}=0, \quad \bar{\alpha}=\frac{-(C+D) \pm \sqrt{(C+D)^{2}-4 D C}}{2}$.

It shows that the deformation is always stable.

(ii) For short wavelength $(\beta \rightarrow \infty)$, Eq. (19) has only one solution, which is

$\bar{\beta} \rightarrow \infty, \quad \bar{\alpha}=-\frac{1}{A}$.

It is again always stable.

But we can see that there is a negative term $1-B+D$ which may lead to instability; it must 
occur at special wave numbers. Therefore, it is of interest to seek the wave number $\bar{\beta}_{\mathrm{m}}$ for which the corresponding $\alpha_{\mathrm{m}}>0$ is a maximum. In addition to the spectral equation (19), $\bar{\alpha}_{\mathrm{m}}$ and $\bar{\beta}_{\mathrm{m}}$ must satisfy the equation

$$
\frac{\mathrm{d} \bar{\alpha}}{\mathrm{d} \bar{\beta}^{2}}=0
$$

which is

$\bar{\beta}_{\mathrm{m}}^{2}=-\frac{(A+1) \bar{\alpha}_{\mathrm{m}}^{2}+(1-B+D) \bar{\alpha}_{\mathrm{m}}}{2\left(A \bar{\alpha}_{\mathrm{m}}+1\right)}$.

Keeping in mind $\bar{\beta}_{\mathrm{m}}^{2}>0$, we arrive at an important inequality to determine the limit of the $\bar{\alpha}_{\mathrm{m}}$ value

$0<\bar{\alpha}_{\mathrm{m}}<\frac{B-D-1}{1+A}=\bar{\alpha}_{\mathrm{m}}^{*}$.

Combining both the spectral equation (19) and the extreme condition (24), the equation to determine $\bar{\alpha}_{\mathrm{m}}$ can then be obtained as

$f_{1}=f_{2}$,

where

$f_{1}=4\left(\bar{\alpha}_{\mathrm{m}}^{3}+(C+D) \bar{\alpha}_{\mathrm{m}}^{2}+D C \bar{\alpha}_{\mathrm{m}}\right)\left(A \bar{\alpha}_{\mathrm{m}}+1\right)$,

$f_{2}=\left[(A+1) \bar{\alpha}_{\mathrm{m}}^{2}+(1-B+D) \bar{\alpha}_{\mathrm{m}}\right]^{2}$.

If the permeability obstruction may be neglected, which means, $D=0$, the same solutions as those of Bai [16] may be obtained and the criterion of instability is as follows from Fig. 1a that for the region $\bar{\alpha}_{\mathrm{m}}>0$. It may be seen that the left branch of function $f_{2}$ and the right branch of $f_{1}$ must have an intersection between 0 and $\bar{\alpha}_{\mathrm{m}}^{*}$ as long as

$B-1>2 \sqrt{C}$.

It is the criterion for the existence of a solution $\bar{\alpha}_{\mathrm{m}}$ and hence is what we desired. In most conditions $C \approx 0$, then the criterion can be simplified to

$B=\frac{C_{1} \tau_{0} Q_{0}}{\rho_{\mathrm{s}} c_{v} R_{0}}>1$

This means the condition of instability is that thermal softening overcomes the strain hardening. It is very interesting that whether instability occurs or not is not related to the obstruction coefficient
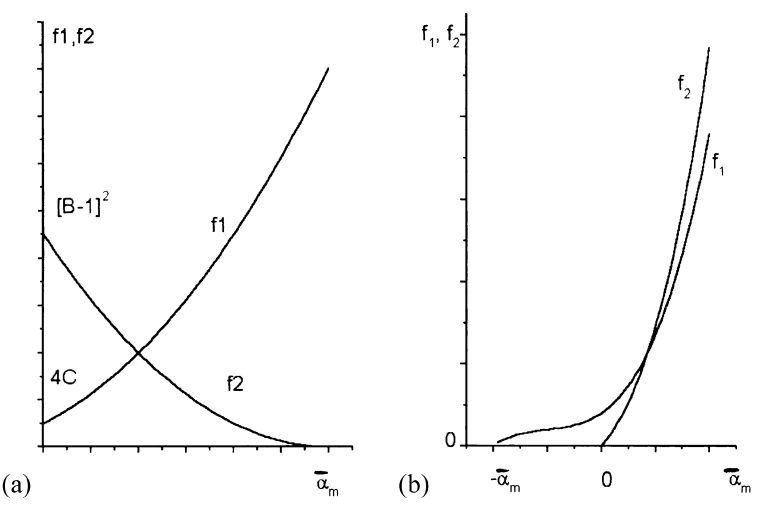

Fig. 1. Plots of the functions $f_{1}, f_{2}$, defined in Eqs. (26) and (27).

$K$ and the strain-rate hardening $H_{0}$. However, these factors influence instability markedly in some other aspects which will be discussed later.

If $D \neq 0$, it can be seem that if only $\partial f_{1} /\left.\partial \bar{\alpha}_{\mathrm{m}}\right|_{\bar{\alpha}_{\mathrm{m}}=0}<\partial f_{2} /\left.\partial \bar{\alpha}_{\mathrm{m}}\right|_{\bar{\alpha}_{\mathrm{m}}=0}$ (Fig. 1b), there is at least one positive $\bar{\alpha}$. It may be expressed as follows:

$B-1-D>\sqrt{4(A D C+C+D)}$.

In most conditions, $\mathrm{A}, C \ll 1$, and the right term in the inequality may be neglected compared with the left term. Then, it has the following form:

$B-D=\frac{C_{1} \tau_{0} Q_{0}-K n_{0}^{2} \lambda}{\rho_{\mathrm{s}} c_{v} R_{0}}>1$.

This means that the condition of instability is that the softening effect caused by thermal and permeability obstruction overcomes the strain hardening effect. The strain hardening effect and the permeability effect are the obstructed factors of localization while the thermal effect is a promoting factor.

The intersection $\bar{\alpha}_{\mathrm{m}}$ in Fig. 1 and the corresponding value of $\bar{\beta}_{\mathrm{m}}$ represent the most probable unstable solution. The solution $\bar{\alpha}_{\mathrm{m}}$ has the same order as $\bar{\alpha}_{\mathrm{m}}^{*}$.

Hence, for qualitative discussion, the value of $\bar{\alpha}_{\mathrm{m}}^{*}$ can be used to represent the point of intersection $\bar{\alpha}_{\mathrm{m}}$. The characteristic time can be expressed as

$$
\begin{aligned}
t_{\mathrm{c}} & \sim \frac{1}{\alpha_{\mathrm{m}}} \sim \frac{\rho_{\mathrm{s}}}{\bar{\alpha}_{\mathrm{m}} K} \sim \frac{\rho_{\mathrm{s}}(A+1)}{K(B-D-1)} \\
& =\frac{\rho_{\mathrm{s}}^{2} c_{v} R_{0}\left(c_{v} H_{0}+\lambda\right)}{K \lambda\left(c_{1} \tau_{0} Q_{0}-K n_{0}^{2} \lambda-\rho_{\mathrm{s}} c_{v} R_{0}\right)} .
\end{aligned}
$$


It is obvious that the characteristic time is affected by strain-rate hardening, permeability obstruction, strain hardening and thermo-softening.

The characteristic length $l_{\mathrm{c}}$ is related to $t_{\mathrm{c}}$ by

$$
l_{\mathrm{c}}^{2} / t_{\mathrm{c}} \sim \alpha_{\mathrm{m}} / \beta_{\mathrm{m}}^{2} \sim \frac{\lambda}{\rho c_{v}}\left(\bar{\beta}_{\mathrm{m}}^{2} / \bar{\alpha}_{\mathrm{m}}\right) \sim a\left(\bar{\beta}_{\mathrm{m}}^{2} / \bar{\alpha}_{\mathrm{m}}\right)
$$

where $a$ is the thermal diffusivity. Therefore, $l_{\mathrm{c}}$ is the pattern length rather than the thermal diffusion length $l_{0}$ which is connected to time $t$ by $l_{0}^{2} / t \sim a$.

Next, the three interesting special cases: no permeability, no strain hardening and no temperature will be discussed.

\section{Some other conditions}

\subsection{Adiabatic conditions, $\lambda=0$}

In this case, the spectral equation (17) becomes

$$
\begin{gathered}
\rho_{\mathrm{s}}^{2} c_{v} \alpha^{2}+\left(\rho_{\mathrm{s}} c_{v} H_{0} \beta^{2}+\rho_{\mathrm{s}} C_{1} \dot{\gamma}_{0} Q_{0}+\rho_{\mathrm{s}} c_{v} K n_{0}^{2}\right) \alpha \\
+K n_{0}^{2} C \dot{\gamma}_{0} Q_{0}+\rho_{\mathrm{s}} c_{v} \beta^{2} R_{0}-C_{1} \tau_{0} Q_{0} \beta^{2}=0 .
\end{gathered}
$$

It $K n_{0}^{2} C \dot{\gamma}_{0} Q_{0}+\rho_{\mathrm{s}} c_{v} \beta^{2} R_{0}-C_{1} \tau_{0} Q_{0} \beta^{2}<0$, namely $B-1-D>0$, it is certain that $\alpha$ has a positive root and the instability must occur. It is appreciable that the same criterion (31) can be used whether the instability is adiabatic or not. But the equation $\left(\mathrm{d}^{2} \alpha / \mathrm{d} \beta^{2}=0\right)$ leads to

$\alpha_{\mathrm{m}}=\frac{R_{0}}{H_{0}}(B-1)$

and

$\beta_{\mathrm{m}} \rightarrow \infty$.

This means the characteristic length approaches zero in the adiabatic case.

\subsection{The effect of permeability}

(i) $K \rightarrow \infty$, means $D \rightarrow \infty$, the spectral equation (17) becomes

$$
\rho_{\mathrm{s}} c_{v} \alpha^{2}+\left(\lambda \beta^{2}+C \dot{\gamma}_{0} Q_{0}\right) \alpha=0 .
$$

$\alpha$ has two solutions

$\alpha=0, \quad \alpha=-\frac{\lambda \beta^{2}+C \dot{\gamma}_{0} Q_{0}}{\rho_{\mathrm{s}} c_{v}}$.

It shows that in this case the deformation is stable. Therefore, the permeability has a negative effect on the emergence of localization deformation of saturated soil.

If $K \rightarrow 0$, which means $D=0$. The condition of instability is the same as Eq. (28), i.e.

$B-1>\sqrt{4 C}$.

We can see, if $C \ll 1$, $\alpha$ will have a positive real root and instability will occur when $B>1$.

\subsection{No strain hardening, $R_{0}=0$} form

Now, the spectral equation (17) will take the next

$\rho_{\mathrm{s}}^{2} c_{v} \alpha^{3}+A_{1} \alpha^{2}+A_{2} \alpha=0$,

where

$A_{1}=\left(\rho_{\mathrm{s}} c_{v} H_{0}+\rho_{\mathrm{s}} \lambda\right) \beta^{2}+\rho_{\mathrm{s}} C_{1} \dot{\gamma}_{0} Q_{0}+\rho_{\mathrm{s}} c_{v} K n_{0}^{2}$,

$A_{2}=\lambda H_{0} \beta^{4}+K n_{0}^{2} \lambda \beta^{2}+K n_{0}^{2} C \dot{\gamma}_{0} Q_{0}-C_{1} \tau_{0} Q_{0} \beta^{2}$.

The condition $\alpha$ has positive real root as follows in this case:

$\lambda H_{0} \beta^{4}+K n_{0}^{2} \lambda \beta^{2}+K n_{0}^{2} C \dot{\gamma}_{0} Q_{0}-C_{1} \tau_{0} Q_{0} \beta^{2}<0$.

In this criterion obstruction coefficient $K$, strainrate hardening $H_{0}$ and thermal softening play the role. The equation $\left(\mathrm{d}^{2} \alpha / \mathrm{d} \beta^{2}=0\right)$ leads to

$\beta_{\mathrm{m}}^{2}=\frac{C_{1} \tau_{0} Q_{0}-K n_{0}^{2} \lambda-\rho_{s}\left(c_{v} H_{0}+\lambda\right) \alpha_{\mathrm{m}}}{2 \lambda H_{0}}$

$0 \leqslant \alpha_{\mathrm{m}} \leqslant \frac{C_{1} \tau_{0} Q_{0}-K n_{0}^{2} \lambda}{\rho_{\mathrm{s}}\left(c_{v} H_{0}+\lambda\right)}$.

Combining Eqs. (42) and (41), we have the following equations for $\alpha_{\mathrm{m}}$ :

$$
\begin{aligned}
4 \lambda & H_{0}\left(c_{v} \alpha_{\mathrm{m}}+\frac{C_{1} \dot{\gamma}_{0} Q_{0}+c_{v} K n_{0}^{2}}{\rho_{\mathrm{s}}}\right) \\
& =\left[\frac{C_{1} \tau_{0} Q_{0}-K n_{0}^{2} \lambda}{\rho_{\mathrm{s}}}-\left(\lambda+c_{v} H_{0}\right) \alpha_{\mathrm{m}}\right]^{2} .
\end{aligned}
$$


There must be a solution for $\alpha_{\mathrm{m}}$ between 0 and $\left(C_{1} \tau_{0} Q_{0}-K n_{0}^{2} \lambda\right) / \rho_{\mathrm{s}}\left(\lambda+c_{v} H_{0}\right)$ satisfying criterion (41).

In this case, the characteristic time is

$t_{\mathrm{c}} \sim \frac{1}{\alpha_{\mathrm{m}}} \sim \frac{\rho_{\mathrm{s}}\left(\lambda+c_{v} H_{0}\right)}{C_{1} \tau_{0} Q_{0}-K n_{0}^{2} \lambda}$.

\subsection{No thermal softening, $Q_{0}=0$}

Now, we turn to discuss the second mode of instability in which there is no thermal softening; in this case, we can formulate the spectral equation

$\rho_{\mathrm{s}}^{2} c_{v} \alpha^{3}+A_{1} \alpha^{2}+A_{2} \alpha+\lambda R_{0} \beta^{4}=0$

where

$A_{1}=\left(\rho_{2} c_{v} H_{0}+\rho_{\mathrm{s}} \lambda\right) \beta^{2}+\rho_{\mathrm{s}} c_{v} K n_{0}^{2}$,

$A_{2}=\lambda H_{0} \beta^{4}+K n_{0}^{2} \lambda \beta^{2}+\rho_{\mathrm{s}} c_{v} \beta^{2} R_{0}$.

Though $H_{0}, K$ must be positive, $R_{0}$ may be negative. Therefore, $R_{0}<0$ may be another possible cause of instability. Eq. (46) can be rewritten in the form

$$
\begin{aligned}
& \rho_{\mathrm{s}} c_{v} \alpha^{3}+A_{1} \beta^{2} \alpha^{2}+\lambda H_{0} \beta^{4}+K n_{0}^{2} \lambda \beta^{2} \alpha \\
& =\rho_{\mathrm{s}} c_{v} \beta^{2}\left|R_{0}\right| \alpha+\lambda\left|R_{0}\right| \beta^{2} .
\end{aligned}
$$

It is easy to see that there must be a solution $\alpha>0$; therefore, deformation must be unstable. It is very simple to show that no maximum in $\alpha$ exists and $\alpha$ is a monotonically increasing function of $\beta$, with

$\lim _{\beta \rightarrow 0} \alpha \rightarrow 0$ and $\lim _{\beta \rightarrow \infty} \alpha \rightarrow \frac{\left|R_{0}\right|}{H_{0}}$

$\lim _{\beta \rightarrow \infty} t=t_{\text {min }}=\frac{H_{0}}{\left|R_{0}\right|}$.

This implies that the shorter the wavelength, the earlier the occurrence of instability. Nevertheless, it is a totally different instability mode. There is no further criterion except $R_{0}<0$, with which, as we have seen, are associated no characteristic length and time but there exists a minimum time $t_{\min }$.

\section{Conclusions}

It has been shown that there may exist two types of possible instability of saturated soil under shear load considering thermal effect. One is dominated by thermal softening while the other by strain softening. The criterion for the first mode of instability combines thermal softening, strain hardening and the other one requires strain softening conditions. This is only a theoretical paper and experiments for testing should be processed afterwards.

\section{References}

[1] J.R. Rice, On the stability of dilatant hardening for saturated rock masses, J. Geophys. Res. 80 (11) (1975) 1531-1536.

[2] I. Vardoulakis, Stability and bifurcation of undrained plane rectilinear deformations on water-saturated granular soils, Int. J. Num. Anal. Methods Geomech. 9 (1985) 399-414.

[3] I. Vardoulakis, Dynamic stability analysis of undrained simple shear on water-saturated soils, Int. J. Num. Anal. Methods Geomech. 10 (1986) 177-190.

[4] B. Loret, J.H. Provest, Dynamic strain localization in fluid-saturated porous media, J. Engng. Mech. ASCE 117 (1991) 907-922.

[5] P.V. Lade, R.B. Nelson, Y.M. Ito, Instability of granular materials with nonassociated flow, J. Engng. Mech. ASCE 114 (1988) 2173-2191.

[6] P.V. Lade, Instability and liquefaction of granular material, Comp. Geotechnics 16 (2) (1994) 123-151.

[7] I. Vardoulakis, B. Graf, Calibration of constitutive models for granular materials using data from biaxial experiments, Geotechnique 35 (1985) 299-317.

[8] S. Pietruszczak, X. Niu, On the description of localized deformation, Int. J. Num. Anal. Methods Geomech. 17 (1993) 791-805.

[9] S. Pietruszczak, Undrained responses of granular soil involving localized deformation, J. Engng. Mech. ASCE 121 (12) (1995) 1292-1297.

[10] T. Hueckel, G. Baldi, Thermoplasticity of saturated clays: experimental constitutive study, J. Geotech. Engng. ASCE 116 (12) (1990) 1778-1796.

[11] T. Hueckel, M. Borsetto, Thermoplasticity of saturated soils and shales: constitutive equations, J. Geotech. Engng. ASCE 116 (12) (1990) 1165-1777.

[12] R.G. Campanella, J.K. Mitchell, Influence of temperature variations of soil behavior, J. Soil Mech. Found. Engng. Div. ASCE 94 (3) (1968) 709-734.

[13] D.S. Drumheller, A. Bedford, A thermomechanical theory for reacting immiscible mixtures, Arch. Rational Mech. Anal. (1980) 257-284.

[14] Yu Shanbing, Steady advance of coal and gas bursts, Acta Mech. Sinica 20 (2) (1988).

[15] C.C. Lin, The theory of hydrodynamic stability, Cambridge University Press, Cambridge, 1995, pp. 1-30.

[16] Y.L. Bai, Thermo-plastic instability in simple shear, J. Mech. Phys. Solids 30 (4) (1982) 195-206. 